\section{Tuberculose entre crianças e adolescentes indígenas no Brasil: fatores associados ao óbito e ao abandono do tratamento}

\author{
Tuberculosis in indigenous children and \\ adolescents in Brazil: factors associated \\ with death and treatment dropout
}

\section{Tuberculosis entre niños y adolescentes indígenas en Brasil: factores asociados al óbito y al abandono del tratamiento}

\section{Resumo}

O objetivo deste estudo foi descrever características clinicas e sociodemográficas, estimar a incidência da tuberculose (TB), além de analisar fatores associados ao abandono e ao óbito na vigência do tratamento dos casos de TB notificados entre crianças e adolescentes indígenas, no Brasil, entre 2006-2016. Realizou-se análise da série histórica de incidência, segundo faixa etária e macrorregião e utilizou-se regressão logística multinomial para elucidar fatores associados ao abandono e ao óbito. Do total de 2.096 casos notificados, $88,2 \%$ tiveram cura, 7,2\% abandonaram o tratamento e 4,6\% evoluiram para óbito. Houve predomínio de casos em meninos de 15-19 anos e maior proporção de óbitos $(55,7 \%) \mathrm{em}<4$ anos. Considerando o conjunto de crianças e adolescentes indígenas com TB no Brasil, a incidência média foi 49,1/100 mil, variando de 21,5/100 mil a 97,6/100 mil nas regiões Nordeste e Centro-oeste, respectivamente. Os casos com acompanhamento insuficiente e regular tiveram maiores chances de abandono (OR = 11,1; IC95\%: 5,2-24,8/ $O R=4,4$; IC95\%: 1,9-10,3) e óbito $(O R=20,3$; IC95\%: 4,9-84,9/OR = 5, 1; IC95\%: 1,2-22,7). Os casos em retratamento (OR = 2,4; IC95\%: 2,08-8,55) e com anti-HIV positivo (OR = 8,2; IC95\%: 2,2-30,9) também mostraram-se associados ao abandono. As formas clínicas extrapulmonar $(O R=1,8$; IC95\%: 1,1-3,3) e mista $(O R=5,6$; IC95\%: 2,8-11,4), os casos em $<4$ anos $(O R=$ 3, 1; IC95\%: 1,5-6,4) e os casos provenientes das regiões Norte (OR = 2,8; IC95\%: 1,1-7,1) e Centro-oeste (OR = 2,8; IC95\%: 1,1-7,0) mostraram-se associados ao óbito. Acreditamos que o controle da TB em crianças e adolescentes indígenas não poderá ser alcançado sem investimentos em pesquisa e desenvolvimento e sem a redução das desigualdades sociais.

Tuberculose; Índios Sul-Americanos; Morte; Pacientes Desistentes do Tratamento
Paulo Victor de Sousa Viana 1

Stefano Barbosa Codenotti 2

Ana Luiza Bierrenbach 3

Paulo Cesar Basta 1

doi: $10.1590 / 0102-311 \times 00074218$

\section{Correspondência}

P. C. Basta

Departamento de Endemias Samuel Pessoa, Escola Nacional de Saúde Pública Sergio Arouca, Fundação Oswaldo Cruz. Rua Leopoldo Bulhões 1480, sala 608, Rio de Janeiro, RJ 21041-210, Brasil.

paulobasta@gmail.com

1 Escola Nacional de Saúde Pública Sergio Arouca, Fundação Oswaldo Cruz, Rio de Janeiro, Brasil.

2 Secretaria de Vigilância em Saúde, Ministério da Saúde, Brasília, Brasil.

3 Instituto de Ensino e Pesquisa, Hospital Sírio-Libanês, São Paulo, Brasil. 


\section{Introdução}

Estudos recentes apontam que a situação de saúde dos povos indígenas no Brasil e na América Latina é precária quando comparada à sociedade envolvente 1,2,3,4,5,6,7,8. Parte dessa disparidade resulta do processo de colonização e se estende até os dias atuais, estando relacionada à perda de autonomia sobre os territórios tradicionais e à discriminação contra a cultura e o conhecimento ancestral 1. Esse processo histórico deixou como legado a pobreza e a marginalização aos povos tradicionais 2,3 e resultou em vultosas desvantagens que se refletem, sobretudo, nos indicadores de saúde, incluindo elevadas taxas de mortalidade infantil, altas prevalências de doenças transmissíveis, emergência de agravos não transmissíveis, violência, além de alarmantes cifras de desnutrição infantil 4,5,6,7,8.

Nesse contexto de desigualdades, a tuberculose (TB) ganha destaque devido às altas taxas de incidência reportadas 9,10,11,12, à elevada concentração de casos ao longo de fronteiras internacionais 10; ao surgimento de resistência aos fármacos 11; às prevalências de infecção latente que atingem quase $50 \%$ da população em algumas etnias 12,13,14; aos padrões de transmissão recente em algumas aldeias 13 e à elevada concentração de casos entre menores de 15 anos 15,16,17.

No que concerne a TB infantil, alguns autores são enfáticos em destacar as dificuldades para um correto diagnóstico, uma vez que as crianças geralmente apresentam sintomas inespecíficos e a confirmação bacteriológica é difícil. A coleta de escarro é particularmente complexa em crianças menores de dez anos. Mesmo que amostras biológicas sejam obtidas, o resultado pode ser falso-negativo 18,19. A fim de enfrentar esse problema, o Ministério da Saúde preconiza que o diagnóstico da TB em crianças seja realizado com base em um sistema de pontuação 20 , validado e com boa acurácia 21 , que considera achados clínicos, história de contato com doentes bacilíferos, vacinação BCG, resultado da prova tuberculínica e achados radiológicos.

Apesar do crescente interesse sobre aspectos epidemiológicos da TB entre populações indígenas, ainda são escassos os estudos entre as crianças e adolescentes no Brasil. Os poucos trabalhos disponíveis na literatura incluem amostras pequenas com representatividade local ou regional 16,22,23. Portanto, pouco se sabe sobre características clínicas de crianças e adolescentes indígenas com TB no Brasil, não há estimativas de incidência para o país como um todo e não existem dados sobre fatores associados aos resultados do tratamento.

Nosso estudo teve como objetivo descrever características clínicas e sociodemográficas, estimar a incidência e a tendência temporal e analisar fatores associados ao abandono e ao óbito na vigência do tratamento entre os casos de TB notificados nas crianças e adolescentes indígenas, no Brasil, ao longo de uma década.

\section{Métodos}

\section{População de estudo}

A população de estudo foi composta pelos casos de TB notificados no Sistema de Informações de Agravos de Notificação (SINAN) entre crianças e adolescentes indígenas, no período de 1o de janeiro de 2006 a 31 de dezembro de 2016, em todo território nacional.

\section{Fonte de dados}

De acordo com o Guia de Vigilância em Saúde 20, os casos detectados na rotina dos serviços devem ser obrigatoriamente notificados no SINAN. Para tal, utilizam-se formulários padronizados: ficha de notificação e boletim de acompanhamento dos casos.

Dentre o conjunto de variáveis presentes na ficha de notificação, encontra-se a variável cor ou raça que deve ser preenchida por autoclassificação, de acordo com as categorias adotadas pelo Instituto Brasileiro de Geografia e Estatística (IBGE), em todas suas pesquisas de demografia e saúde: branca, preta, amarela, parda e indígena. Em tese, no momento do preenchimento da ficha de notificação os profissionais de saúde devem perguntar ao doente como ele se classifica nas categorias acima mencionadas. 
No período de estudo foram notificados 952.420 casos de TB no Brasil. Desses, $10.051(1,1 \%)$ foram em indígenas. Considerando somente crianças e adolescentes de 0-19 anos, houve $2.349(2,9 \%)$ em indígenas e 79.128 (97,1\%) em não indígenas. Para esta análise foram excluídos os casos em que não havia informação na variável situação de encerramento, incluindo a categoria "transferência" para outra unidade de saúde e os casos encerrados como "tuberculose droga resistente (TBDR)". Esses últimos são encaminhados para tratamento e acompanhamento em unidades de referência especializadas e transferidos para o Sistema de Tratamento Especial da Tuberculose (SITE-TB). Somente em consulta ao SITE-TB após um período mínimo de 18 a 24 meses de seguimento é que se pode conhecer a real situação de encerramento de um caso resistente às drogas. Por fim, foram excluídos os casos em que não havia informação sobre sexo, restando 2.096 casos para análise neste estudo.

\section{Variáveis independentes}

\section{- Sociodemográficas}

Foram analisadas as seguintes variáveis: sexo (feminino, masculino); faixa etária (0-4 anos, 5-9 anos, 10-14 anos e 15-19 anos); macrorregião (Norte, Nordeste, Sudeste, Sul e Centro-oeste); e zona de residência (urbana, periurbana, rural e ignorada).

\section{- Clínicas}

As variáveis clínicas exploradas foram: forma clínica (pulmonar, extrapulmonar e mista); radiografia de tórax (suspeito, normal, outra patologia, não realizado); teste tuberculínico (reator forte, reator fraco, não reator, não realizado e ignorado); baciloscopia de escarro (positivo, negativo, não realizado e não se aplica); cultura de escarro e sorologia anti-HIV (positivo, negativo, em andamento e não realizado). Analisou-se ainda a variável tipo de entrada do caso no SINAN (caso novo, reingresso pós-abandono, recidiva e transferência).

Empregou-se um sistema de classificação empírico utilizado por outros autores 10,24,25,26 para análise do acompanhamento dos casos. Este sistema combina cinco recomendações da Sociedade Brasileira de Pneumologia e Tisiologia (SBPT) 27, a saber: (1) se o caso notificado realizou baciloscopia de controle no 2 o mês de tratamento; (2) baciloscopia de controle no 4o mês de tratamento; (3) baciloscopia de controle no 60 mês de tratamento; (4) se houve registro de exames de contatos; e (5) se o tratamento foi diretamente observado.

Nos casos em que não foram seguidas nenhuma ou apenas uma das recomendações acima citadas, o acompanhamento foi classificado como insuficiente. Nos casos em que houve registro de duas recomendações, o acompanhamento foi classificado como regular. Nos casos em que houve registro de três recomendações, o acompanhamento foi considerado como bom. Por fim, nos casos em que foram registradas pelo menos quatro das recomendações, o acompanhamento foi classificado como excelente.

\section{Variável dependente}

Analisou-se como variável dependente a situação de encerramento dos casos, considerando somente as categorias cura, abandono e óbito na vigência do tratamento.

\section{Análise dos dados}

Seguindo as recomendações do Programa Nacional de Controle da Tuberculose (PNCT), para o cálculo dos coeficientes de incidência, utilizou-se no numerador o número de "casos novos" de TB, somados aos casos notificados como tipo de entrada "não sabe" e "pós óbito" entre crianças e adolescentes indígenas, estratificados por faixa etária (0-4 anos, 5-9 anos, 10-14 anos e 15-19 anos) e macrorregião (Norte, Nordeste, Sudeste, Sul e Centro-oeste), no período de 2006 a 2016. No denominador, foi utilizada a população sob risco durante o mesmo período, multiplicado por 100 mil habitantes. 
A variável cor ou raça, de acordo com as categorias adotadas pelo IBGE, foi introduzida nos sistemas de informação gerenciados pelo Ministério da Saúde a partir do ano 2000, pela Portaria no 3.947/199928. Nos instrumentos de notificação do SINAN, as informações sobre cor ou raça passaram a ser inseridas somente a partir de 2001. Todavia, até o ano de 2005 a completude dessa variável era ruim e o preenchimento inferior a $11 \%$, inviabilizando uma análise epidemiológica mais robusta e representativa da população indígena. Dessa forma, optou-se por incluir nesta análise os dados disponíveis no período entre 2006-2016, pois o preenchimento da variável foi superior a $92 \%$.

Para definir a base populacional empregada no cálculo dos coeficientes de incidência foram utilizados os dados dos censos nacionais conduzidos pelo IBGE em 2000 e 2010. Pelo fato de não haver estimativas intercensitárias considerando as diferentes categorias de cor ou raça, utilizou-se taxas médias anuais de crescimento 29, por meio de interpolação geométrica para os anos de 2006, 2007, 2008 e 2009 e por extrapolação para os anos de 2011 a 2016 das crianças e adolescentes indígenas.

Para isso, foram calculadas as taxas geométricas de crescimento anual para cada categoria de faixa etária e macrorregião, utilizando-se a fórmula:

$$
\alpha=\{[(\beta 2 / \beta 1) 1 / 10]-1\}
$$

onde $\alpha$ representa a taxa geométrica anual de crescimento, $\beta 1$ é a população recenseada em 2000 e $\beta 2$ é a população em 2010. Após calcular as taxas de crescimento populacional para cada categoria de faixa etária foram estimadas as populações de 2006 a 2009 e 2011 a 2016 em cada macrorregião. Por exemplo, para obter a população em 2006 foi realizado o seguinte cálculo:

$$
\text { P2006 }=\beta 1(1+\alpha)^{t}
$$

onde $t$ corresponde ao intervalo de tempo entre o anos 2000 e 200629.

Realizamos análises descritivas das características clínicas e sociodemográficas, considerando os desfechos cura, abandono e óbito. Também analisamos as variáveis clínicas, de acordo com faixa etária. Os testes qui-quadrado $\left(\chi^{2}\right)$ e exato de Fisher foram utilizados para a comparação entre as proporções e a variável dependente. Considerou-se o nível de significância $<0,05$.

A regressão logística multinomial 30 foi utilizada para determinar a associação entre as variáveis clínicas e sociodemográficas e a situação de encerramento dos casos. A categoria "cura" foi utilizada como referência para a variável dependente, sendo comparada com as categorias abandono e óbito. “Óbitos por TB" e "óbitos por outras causas" foram considerados um único evento, doravante denominado óbito. A categoria transferência da variável "tipo de entrada” foi excluída da modelagem e as categorias reingresso pós-abandono e recidiva foram consideradas como retratamento. As covariáveis que apresentaram significância estatística na análise descritiva foram incorporadas nos modelos de regressão logística multinomial múltipla. Permaneceram no modelo final aquelas covariáveis que se mostraram significativas ao nível $5 \%$ no teste de Wald.

O teste de Hosmer-Lemeshow 31 foi usado para verificar o ajuste do modelo. Utilizou-se como medida de associação a odds ratio (OR), juntamente com seus intervalos de 95\% de confiança (IC95\%).

O software Microsoft Excel 2016 (https://products.office.com/) foi utilizado para construir os bancos de dados e gerar os gráficos. As análises foram realizadas no software R versão 3.3.3, pacotes "nnet" e "generalhoslem" (http://www.r-project.org).

\section{Aspectos éticos}

Os dados utilizados neste estudo são de acesso público e foram disponibilizados pelo Ministério da Saúde via PNCT por meio do Sistema Eletrônico do Serviço de Informação ao Cidadão (e-SIC; https://esic.cgu.gov.br/sistema/site/index.aspx), com fundamentação na Lei de Acesso à Informação 32, sob o protocolo no 25820.000870/2018-93. O estudo foi aprovado pelo Comitê de Ética em Pesquisa da Escola Nacional de Saúde Pública Sergio Arouca/Fundação Oswaldo Cruz (CEP/ENSP), sob parecer CAAE: 14643713.0.0000.5240.

Ressalta-se que não houve identificação nominal, tampouco contato entre os pesquisadores e os sujeitos investigados. 


\section{Resultados}

O coeficiente médio de incidência de TB para crianças e adolescentes indígenas foi estimado em 49,1 casos por 100 mil habitantes (todas as formas), no período de 2006 a 2016. A maior incidência para o país foi registrada em 2015 (61,8/100 mil) e a menor em 2006 (41,1/100 mil), com tendência de aumento das notificações ao longo do período (Figura 1).

Todavia, a análise da incidência por macrorregião e faixa etária revelou desigualdades expressivas entre as regiões brasileiras. Na Região Centro-oeste concentraram-se as maiores incidências do país no período (97,6/100 mil habitantes). Em seguida, aparecem as regiões Sudeste (84,5/100 mil), Norte (42,4/100 mil), Sul (24,3/100 mil) e Nordeste (21,5/100 mil) (Figura 1 e Material Suplementar, Tabela S1, http://cadernos.ensp.fiocruz.br/site/public_site/arquivo/suppl-e00074218_6918.pdf).

$\mathrm{Na}$ análise da série histórica, no ano de 2015 , foram registradas as maiores incidências nas regiões Sudeste e Centro-oeste (170,3 e 168,4/100 mil, respectivamente). Já as menores incidências foram observadas nas regiões Nordeste e Sudeste no ano de 2006 (15,1 e 8,9/100 mil, respectivamente) (Figura 1 e Material Suplementar, Tabela S1, http://cadernos.ensp.fiocruz.br/site/public_site/arqui vo/suppl-e00074218_6918.pdf).

Excetuando-se os anos de 2006 e 2007 e a Região Sul do país, as maiores incidências concentraram-se nos adolescentes na faixa etária de 15-19 anos em todas as macrorregiões e em praticamente todo o período analisado (Figura 1 e Material Suplementar, Tabela S1, http://cadernos.ensp.fio cruz.br/site/public_site/arquivo/suppl-e00074218_6918.pdf). Na Região Sul, as maiores incidências foram registradas entre as crianças de 0-4 anos. Por outro lado, as menores incidências foram observadas entre as crianças de 5-9 anos, em praticamente todas as macrorregiões (Figura 1).

De acordo com a situação de encerramento, do total de 2.096 casos notificados, $88,2 \%$ evoluíram para cura, 7,2\% abandonaram o tratamento e 4,6\% foram a óbito (Tabela 1). Destaca-se o encerramento de um caso por TBDR, numa criança de 0-4 anos, que apresentava forma clínica pulmonar e residia na zona urbana da Região Centro-oeste. Constatou-se que o adoecimento por TB foi ligeiramente mais frequente entre os meninos $(53,1 \%)$, com uma razão de casos de 1,1 masculino/1,0 feminino (Tabela 1).

Houve maiores proporções de abandono entre as meninas $(8,2 \%)$. Os óbitos foram discretamente mais frequentes entre os meninos (5\%) e na faixa etária de 0-4 anos ( $8,8 \%)$. Os maiores percentuais de cura foram observados nos indígenas de 10-14 anos (91,4\%). (Tabela 1).

Mais de um terço $(39,4 \%)$ dos casos e as maiores proporções de óbito $(5,7 \%)$ foram notificados na Região Norte. A maioria das notificações (68,5\%) concentrou-se na zona rural do país (Tabela 1).

A forma clínica pulmonar foi mais frequente $(83,3 \%)$ no conjunto das notificações, variando entre $89,6 \%$ e 3,6\% nos casos que evoluíram para cura e óbito, respectivamente. Chama atenção a disparidade nas notificações da forma mista entre os óbitos (19,2\%), e os casos que evoluíram para cura (76,7\%) e que abandonaram o tratamento (4,1\%). A maior proporção de abandono concentrou-se entre os casos extrapulmonares $(10,1 \%)$ (Tabela 2$)$.

As formas extrapulmonares e mistas mais frequentes foram: ganglionar periférica $(n=151 ; 43 \%)$, pleural $(n=64 ; 18,2 \%)$, outras $(n=53 ; 15,1 \%)$, miliar $(n=33 ; 9,4 \%)$, meníngea $(n=27 ; 7,7 \%)$, óssea $(\mathrm{n}=14 ; 4 \%)$, cutânea $(\mathrm{n}=5 ; 1,4 \%)$, laríngea $(\mathrm{n}=2 ; 0,6 \%)$, genitourinária e ocular $(\mathrm{n}=1 ; 0,3 \%)$ (dados não apresentados).

Analisando as características clínicas segundo faixa etária, observou-se maior frequência de formas pulmonares entre os adolescentes (87,9\%) e extrapulmonar na faixa etária de 5-9 anos (20,9\%). Uma maior concentração de reações fortes ao teste tuberculínico entre as crianças de 5-9 anos (26\%) e maior proporção de não realização entre os adolescentes (79,7\%). Na faixa etária de 10-14 anos foram observadas as maiores positividades na baciloscopia de escarro $(29,7 \%)$ e menores proporções de não realização deste exame. Resultados positivos na cultura de escarro foram mais frequentes entre os adolescentes de 15-19 anos (18,9\%) (Tabela 3).

Em relação aos exames empregados para o diagnóstico, em 319 notificações $(15,2 \%)$ a radiografia de tórax não foi realizada, em 139 (6,6\%) os laudos foram normais, e em 23 (1,1\%) o exame radiológico foi indicativo de outra patologia. Em suma, quase um quarto $(22,9 \%)$ dos casos que foram submetidos a tratamento para TB no período não continham dados radiológicos conclusivos. Não houve diferença estatisticamente significativa, considerando a situação de encerramento (Tabela 2). 
Figura 1

Coeficientes de incidência de tuberculose, todas as formas, por 100 mil habitantes. Brasil e macrorregiões, 2006 a 2016.
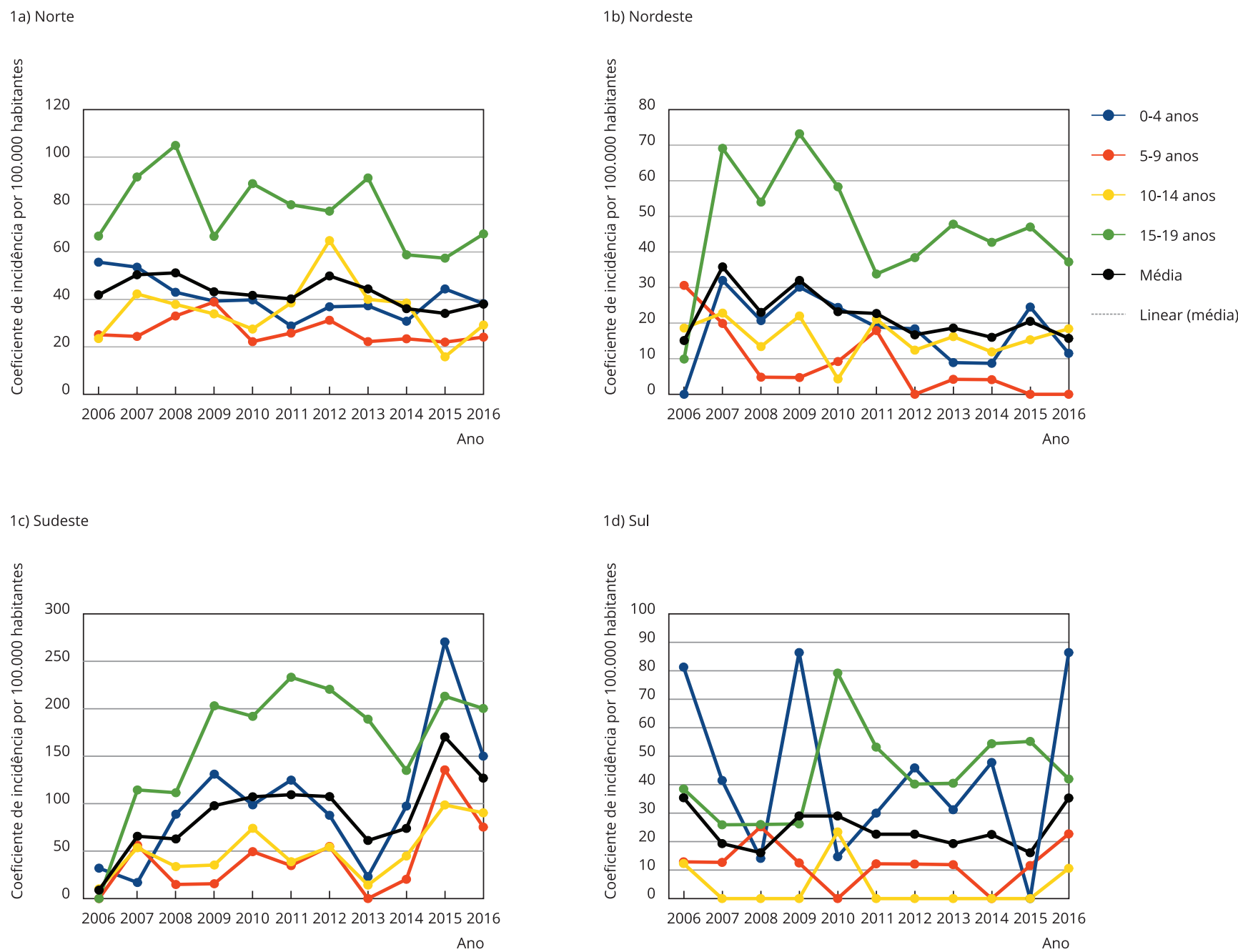

1e) Centro-oeste

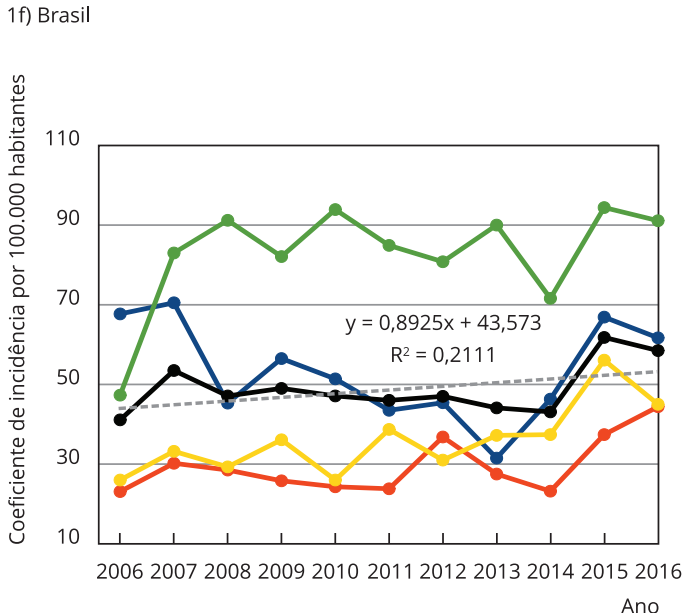


Tabela 1

Distribuição dos casos de tuberculose entre crianças indígenas segundo encerramento e variáveis sociodemográficas. Brasil, 2006 a 2016.

\begin{tabular}{|c|c|c|c|c|c|c|c|c|c|}
\hline \multirow[t]{2}{*}{ Características } & \multicolumn{2}{|c|}{ Total * } & \multicolumn{2}{|c|}{ Cura ** } & \multicolumn{2}{|c|}{ Abandono ** } & \multicolumn{2}{|c|}{ Óbito ** } & \multirow[t]{2}{*}{ Valor de $p$} \\
\hline & $\mathbf{n}$ & $\%$ & $\mathbf{n}$ & $\%$ & $\mathbf{n}$ & $\%$ & $\mathbf{n}$ & $\%$ & \\
\hline Sexo & & & & & & & & & 0,153 \\
\hline Masculino & 1.112 & 53,1 & 973 & 88,9 & 91 & 6,1 & 48 & 5,0 & \\
\hline Feminino & 984 & 46,9 & 875 & 87,5 & 60 & 8,2 & 49 & 4,3 & \\
\hline Faixa etária (anos) & & & & & & & & & $<0,001$ \\
\hline $0-4$ & 611 & 29,1 & 526 & 86,1 & 31 & 5,1 & 54 & 8,8 & \\
\hline $5-9$ & 335 & 16,1 & 295 & 88,1 & 26 & 7,8 & 14 & 4,2 & \\
\hline $10-14$ & 397 & 18,9 & 363 & 91,4 & 24 & 6,0 & 10 & 2,5 & \\
\hline $15-19$ & 753 & 35,9 & 664 & 88,2 & 70 & 9,3 & 19 & 2,5 & \\
\hline Macrorregião & & & & & & & & & $<0,001$ \\
\hline Norte & 826 & 39,4 & 727 & 88,0 & 52 & 6,3 & 47 & 5,7 & \\
\hline Nordeste & 210 & 10,0 & 174 & 82,9 & 31 & 14,8 & 5 & 2,4 & \\
\hline Sudeste & 239 & 11,4 & 205 & 85,8 & 28 & 11,7 & 6 & 2,5 & \\
\hline Sul & 83 & 4,0 & 72 & 86,7 & 8 & 9,6 & 3 & 3,6 & \\
\hline Centro-oeste & 738 & 35,2 & 670 & 90,8 & 32 & 4,3 & 36 & 4,9 & \\
\hline Zona de residência & & & & & & & & & 0,005 \\
\hline Urbana & 374 & 17,9 & 316 & 84,5 & 35 & 9,4 & 23 & 6,1 & \\
\hline Rural & 1.436 & 68,5 & 1.290 & 89,8 & 85 & 5,9 & 61 & 4,2 & \\
\hline Periurbana & 20 & 0,9 & 19 & 95,0 & 0 & 0,0 & 1 & 5,0 & \\
\hline Ignorada & 266 & 12,7 & 223 & 83,8 & 31 & 11,7 & 12 & 4,5 & \\
\hline Total & 2.096 & 100,0 & 1.848 & 88,2 & 151 & 7,2 & 97 & 4,6 & \\
\hline
\end{tabular}

* Valores percentuais calculados pelo total na coluna;

** Valores percentuais calculados pelo total na linha.

O teste tuberculínico não foi realizado em $71,7 \%$ do total de casos que iniciaram tratamento, variando de 70,6\% a 81,4\%, nos casos que evoluíram para cura e óbito, respectivamente (dados não apresentados). Houve maior concentração de reações $\geq 10 \mathrm{~mm}$ nos casos que evoluíram para cura $(92,7 \%)$ e menor entre os óbitos $(1,1 \%)$. Por outro lado, a ausência de resposta ao TT foi mais frequente entre os óbitos (5,9\%) (Tabela 2).

A baciloscopia de escarro não foi realizada em $45,1 \%$ das notificações, sendo mais frequente a não realização entre os casos que evoluíram a óbito (6,8\%) (Tabela 2). Dentre os casos que realizaram este exame $(\mathrm{n}=1.106)$, a positividade foi $55 \%$. Ao analisar a positividade, de acordo com a situação de encerramento, observa-se que nos casos em que houve cura a positividade foi 54,4\% (544/1.000). Nos casos que abandonaram o tratamento a positividade foi 64,5\% (49/76) e nos casos que evoluíram para óbito, a positividade foi 50\% (15/15) (dados não apresentados).

A cultura de escarro não foi realizada em 79,5\% das notificações, sendo mais frequente a não realização entre os óbitos $(89,7 \%)$. Dentre os casos que realizaram este exame ( $\mathrm{n}=429)$, a positividade foi $52,1 \%$. Considerando a situação de encerramento, a positividade variou de 51,5\% (203/394) nos casos em que houve cura, a 60\% (15/25) nos casos que abandonaram o tratamento, e $60 \%(6 / 10)$ nos casos que foram a óbito (dados não apresentados).

Em 40,7\% das notificações não foi realizada a sorologia para HIV, sendo mais frequente a não realização entre os casos que evoluíram para óbito (6,6\%). Quanto aos resultados do exame, nota-se uma maior proporção de exames sororreagentes entre os casos de abandono (31,2\%). É importante ressaltar que os resultados negativos foram mais frequentes entre os casos de cura (92\%) (Tabela 2).

Observa-se que elevadas proporções de acompanhamento insuficiente foram registradas entre os óbitos (94\%) e entre os casos que abandonaram o tratamento (13,5\%). Somente $0,4 \%$ dos casos que 


\section{Tabela 2}

Distribuição dos casos de tuberculose entre crianças indígenas segundo encerramento e variáveis clínicas. Brasil, 2006 a 2016.

\begin{tabular}{|c|c|c|c|c|c|c|c|c|c|}
\hline \multirow[t]{2}{*}{ Características } & \multicolumn{2}{|c|}{ Total * } & \multicolumn{2}{|c|}{ Cura ** } & \multicolumn{2}{|c|}{ Abandono ** } & \multicolumn{2}{|c|}{ Óbito ** } & \multirow[t]{2}{*}{ Valor de $p$} \\
\hline & $\mathbf{n}$ & $\%$ & $\mathrm{n}$ & $\%$ & $\mathbf{n}$ & $\%$ & $\mathbf{n}$ & $\%$ & \\
\hline Forma clínica & & & & & & & & & $<0,001$ \\
\hline Pulmonar & 1.745 & 83,3 & 1.563 & 89,6 & 120 & 6,9 & 62 & 3,6 & \\
\hline Extrapulmonar & 278 & 13,2 & 229 & 82,4 & 28 & 10,1 & 21 & 7,6 & \\
\hline Mista & 73 & 3,5 & 56 & 76,7 & 3 & 4,1 & 14 & 19,2 & \\
\hline Raio X & & & & & & & & & 0,793 \\
\hline Suspeito & 1.615 & 77,1 & 1.421 & 88,0 & 120 & 7,4 & 74 & 4,6 & \\
\hline Normal & 139 & 6,6 & 125 & 89,9 & 8 & 5,8 & 6 & 4,3 & \\
\hline Outra patologia & 23 & 1,1 & 21 & 91,3 & 0 & 0,0 & 2 & 8,7 & \\
\hline Não realizado & 319 & 15,2 & 281 & 88,1 & 23 & 7,2 & 15 & 4,7 & \\
\hline Teste tuberculínico & & & & & & & & & 0,004 \\
\hline Reator forte & 355 & 16,9 & 329 & 92,7 & 22 & 6,2 & 4 & 1,1 & \\
\hline Reator fraco & 53 & 2,5 & 49 & 92,5 & 1 & 1,9 & 3 & 5,7 & \\
\hline Não reator & 185 & 8,8 & 166 & 89,7 & 8 & 4,3 & 11 & 5,9 & \\
\hline Não realizado & 1.503 & 71,7 & 1.304 & 86,8 & 120 & 8,0 & 79 & 5,3 & \\
\hline Baciloscopia & & & & & & & & & $<0,001$ \\
\hline Positivo & 608 & 29,0 & 544 & 89,5 & 49 & 8,1 & 15 & 2,5 & \\
\hline Negativo & 498 & 23,8 & 456 & 91,6 & 27 & 5,4 & 15 & 3,0 & \\
\hline Não realizado & 946 & 45,1 & 809 & 88,6 & 73 & 4,5 & 64 & 6,8 & \\
\hline Não se aplica & 44 & 2,1 & 39 & 85,5 & 2 & 7,7 & 3 & 6,8 & \\
\hline Cultura de escarro & & & & & & & & & 0,074 \\
\hline Positivo & 224 & 10,7 & 203 & 90,6 & 15 & 6,7 & 6 & 2,7 & \\
\hline Negativo & 154 & 7,3 & 145 & 94,2 & 8 & 5,2 & 1 & 0,6 & \\
\hline Em andamento & 51 & 2,4 & 46 & 90,2 & 2 & 3,9 & 3 & 5,9 & \\
\hline Não realizado & 1.667 & 79,5 & 1.454 & 87,2 & 126 & 7,6 & 87 & 5,2 & \\
\hline Sorologia para HIV & & & & & & & & & $<0,001$ \\
\hline Positivo & 16 & 0,8 & 9 & 56,2 & 5 & 31,2 & 2 & 12,5 & \\
\hline Negativo & 1.165 & 55,6 & 1.072 & 92,0 & 55 & 4,7 & 38 & 3,3 & \\
\hline Em andamento & 61 & 2,9 & 55 & 90,2 & 5 & 8,2 & 1 & 1,6 & \\
\hline Não realizado & 854 & 40,7 & 712 & 83,4 & 86 & 10,1 & 56 & 6,6 & \\
\hline Tipo de entrada & & & & & & & & & $<0,001$ \\
\hline Caso novo & 1.908 & 91,0 & 1.688 & 88,5 & 126 & 6,6 & 94 & 4,9 & \\
\hline Reingresso & 44 & 2,1 & 27 & 61,4 & 17 & 38,6 & 0 & 0,0 & \\
\hline Recidiva & 35 & 1,7 & 34 & 97,1 & 0 & 0,0 & 1 & 2,9 & \\
\hline Transferência & 109 & 5,2 & 99 & 90,8 & 8 & 7,3 & 2 & 1,8 & \\
\hline Indicador de acompanhamento & & & & & & & & & $<0,001$ \\
\hline Insuficiente & 784 & 30,3 & 604 & 77,0 & 106 & 13,5 & 74 & 9,4 & \\
\hline Regular & 637 & 37,0 & 587 & 92,2 & 32 & 5,0 & 18 & 2,8 & \\
\hline Bom & 222 & 10,9 & 213 & 95,9 & 6 & 2,7 & 3 & 1,4 & \\
\hline Excelente & 453 & 21,7 & 444 & 98,0 & 7 & 1,5 & 2 & 0,4 & \\
\hline Total & 2.096 & 100,0 & 1.848 & 88,2 & 151 & 7,2 & 97 & 4,6 & \\
\hline
\end{tabular}

* Valores percentuais calculado pelo total na coluna;

** Valores percentuais calculado pelo total na linha. 
Tabela 3

Distribuição dos casos de tuberculose entre crianças indígenas segundo faixa etária e variáveis clínicas. Brasil, 2006 a 2016.

\begin{tabular}{|c|c|c|c|c|c|c|c|c|c|c|c|}
\hline \multirow[t]{3}{*}{ Características } & \multicolumn{10}{|c|}{ Faixa etária (anos) } & \multirow[t]{3}{*}{ Valor de $p$} \\
\hline & \multicolumn{2}{|c|}{$0-4$} & \multicolumn{2}{|c|}{$5-9$} & \multicolumn{2}{|c|}{$10-14$} & \multicolumn{2}{|c|}{$15-19$} & \multicolumn{2}{|c|}{ Total } & \\
\hline & $\mathbf{n}$ & $\%$ & $\mathbf{n}$ & $\%$ & $\mathbf{n}$ & $\%$ & $\mathbf{n}$ & $\%$ & $\mathbf{n}$ & $\%$ & \\
\hline \multicolumn{12}{|l|}{ Forma clínica } \\
\hline Pulmonar & 504 & 82,5 & 254 & 75,8 & 325 & 81,9 & 662 & 87,9 & 1.745 & 83,3 & $<0,001$ \\
\hline Extrapulmonar & 82 & 13,4 & 70 & 20,9 & 58 & 14,6 & 68 & 9,0 & 278 & 13,3 & \\
\hline Mista & 25 & 4,1 & 11 & 3,3 & 14 & 3,5 & 23 & 3,1 & 73 & 3,5 & \\
\hline Raio X & & & & & & & & & & & $<0,001$ \\
\hline Suspeito & 495 & 81,0 & 250 & 74,6 & 300 & 75,6 & 570 & 75,7 & 1.615 & 77,1 & \\
\hline Normal & 40 & 6,5 & 28 & 8,4 & 37 & 9,3 & 34 & 4,5 & 139 & 6,6 & \\
\hline Outra patologia & 3 & 0,5 & 9 & 2,7 & 4 & 1,0 & 7 & 0,9 & 23 & 1,1 & \\
\hline Não realizado & 73 & 11,9 & 48 & 14,3 & 56 & 14,1 & 142 & 18,9 & 319 & 15,2 & \\
\hline Teste tuberculínico & & & & & & & & & & & $<0,001$ \\
\hline Reator forte & 87 & 14,2 & 87 & 26,0 & 73 & 18,4 & 108 & 14,3 & 355 & 16,9 & \\
\hline Reator fraco & 20 & 3,3 & 10 & 3,0 & 9 & 2,3 & 14 & 1,9 & 53 & 2,5 & \\
\hline Não reator & 87 & 14,2 & 37 & 11,0 & 30 & 7,6 & 31 & 4,1 & 185 & 8,8 & \\
\hline Não realizado & 417 & 68,2 & 201 & 60,0 & 285 & 71,8 & 600 & 79,7 & 1.503 & 71,7 & \\
\hline Baciloscopia & & & & & & & & & & & $<0,001$ \\
\hline Positivo & 43 & 7,0 & 41 & 12,2 & 118 & 29,7 & 406 & 12,2 & 608 & 29,0 & \\
\hline Negativo & 100 & 16,4 & 83 & 24,8 & 120 & 30,2 & 195 & 24,8 & 498 & 23,8 & \\
\hline Não realizado & 450 & 73,6 & 199 & 59,4 & 154 & 38,8 & 143 & 59,4 & 946 & 45,1 & \\
\hline Não se aplica & 18 & 2,9 & 12 & 3,6 & 5 & 1,3 & 9 & 3,6 & 44 & 2,1 & \\
\hline Cultura de escarro & & & & & & & & & & & $<0,001$ \\
\hline Positivo & 16 & 2,6 & 23 & 6,9 & 43 & 10,8 & 142 & 18,9 & 224 & 10,7 & \\
\hline Negativo & 25 & 4,1 & 14 & 4,2 & 43 & 10,8 & 72 & 4,2 & 154 & 7,3 & \\
\hline Em andamento & 16 & 2,6 & 8 & 2,4 & 11 & 2,8 & 16 & 2,4 & 51 & 2,4 & \\
\hline Não realizado & 554 & 90,7 & 290 & 86,6 & 300 & 75,6 & 523 & 86,6 & 1.667 & 79,5 & \\
\hline Sorologia para HIV & & & & & & & & & & & $<0,001$ \\
\hline Positivo & 6 & 1,0 & 3 & 0,9 & 0 & 0,0 & 7 & 0,9 & 16 & 0,8 & \\
\hline Negativo & 266 & 43,5 & 164 & 49,0 & 240 & 60,5 & 495 & 49,0 & 1.165 & 55,6 & \\
\hline Em andamento & 17 & 2,8 & 9 & 2,7 & 9 & 2,3 & 26 & 2,7 & 61 & 2,9 & \\
\hline Não realizado & 322 & 52,7 & 159 & 47,5 & 148 & 37,3 & 225 & 47,5 & 854 & 40,7 & \\
\hline Total & 611 & 29,2 & 335 & 16,0 & 397 & 18,9 & 753 & 35,9 & 2.096 & 100,0 & \\
\hline
\end{tabular}

foram a óbito e 1,5\% dos casos que abandonaram tiveram acompanhamento excelente. Em contrapartida 98\% dos casos que evoluíram para cura tiveram acompanhamento excelente (Tabela 2).

Os casos que tiveram acompanhamento insuficiente $(\mathrm{OR}=11,1$; IC95\%: 5,2-24,8) e acompanhamento regular $(\mathrm{OR}=4,4$; IC95\%: 1,9-10,3), assim como os casos em retratamento (OR = 2,4; IC95\%: 2,08-8,55), e aqueles com anti-HIV positivo (OR = 8,2; IC95\%: 2,2-30,9) e sorologia não realizada $(\mathrm{OR}=2,2$; IC95\%: 1,5-3,3) tiveram maiores chances de abandono do tratamento. Por outro lado, os casos provenientes da Região Centro-oeste ( $\mathrm{OR}=0,5$; IC95\%: 0,3-0,9) apresentaram menor chance de abandono (Tabela 4).

De modo semelhante, nos casos com acompanhamento insuficiente (OR = 20,3; IC95\%: 4,9-84,9) e regular $(\mathrm{OR}=5,1$; IC95\%: 1,2-22,7), naqueles em que foram notificadas formas extrapulmonares $(\mathrm{OR}=1,8$; IC95\%: 1,1-3,3) e mistas $(\mathrm{OR}=5,6$; IC95\%: 2,8-11,4), entre as crianças de 0-4 anos $(\mathrm{OR}=$ 3,1; IC95\%: 1,5-6,4), nos provenientes das regiões Norte (OR = 2,8; IC95\%: 1,1-7,1) e Centro-oeste $(\mathrm{OR}=2,8$; IC95\%: 1,1-7,0) e naqueles com sorologia anti-HIV não realizada (OR = 1,8; IC95\%: 1,1-2,9), 
Tabela 4

Modelo final de regressão logística multinomial múltipla dos fatores associados ao abandono e ao óbito entre crianças e adolescentes indígenas. Brasil, 2006 a 2016 ( N = 1.987).

\begin{tabular}{|c|c|c|c|c|c|c|}
\hline \multirow[t]{2}{*}{ Fatores de riscos } & \multicolumn{3}{|c|}{ Abandono vs. cura } & \multicolumn{3}{|c|}{ Óbito vs. cura } \\
\hline & OR ajustada & IC95\% & Valor de $p$ * & OR ajustada & IC95\% & Valor de $p$ * \\
\hline \multicolumn{7}{|l|}{ Faixa etária (anos) } \\
\hline $10-14$ & 1,0 & & & 1,0 & & \\
\hline $0-4$ & 0,7 & $0,4-1,3$ & 0,328 & 3,1 & $1,5-6,4$ & 0,002 \\
\hline $5-9$ & 1,1 & $0,6-2,1$ & 0,762 & 1,6 & $0,7-3,8$ & 0,297 \\
\hline 15-19 & 1,7 & $0,9-2,8$ & 0,075 & 1,5 & $0,7-3,4$ & 0,325 \\
\hline \multicolumn{7}{|l|}{ Região } \\
\hline Sudeste & 1,0 & & & & & \\
\hline Norte & 0,6 & 0,$4 ; 1,1$ & 0,088 & 2,8 & $1,1-7,1$ & 0,027 \\
\hline Nordeste & 1,1 & $0,6-2,0$ & 0,801 & 1,3 & $0,3-4,6$ & 0,705 \\
\hline Sul & 0,8 & 0,$3 ; 2,1$ & 0,649 & 1,4 & $0,3-6,4$ & 0,633 \\
\hline Centro-oeste & 0,5 & 0,$3 ; 0,9$ & 0,043 & 2,8 & $1,1-7,0$ & 0,034 \\
\hline \multicolumn{7}{|l|}{ Forma clínica } \\
\hline Pulmonar & 1,0 & & & 1,0 & & \\
\hline Extrapulmonar & 1,1 & $0,7-1,9$ & 0,619 & 1,8 & $1,1-3,3$ & 0,035 \\
\hline Mista & 0,7 & $0,2-2,4$ & 0,584 & 5,6 & $2,8-11,4$ & $<0,001$ \\
\hline \multicolumn{7}{|l|}{ Teste tuberculínico } \\
\hline Não reator & 1,0 & & & 1,0 & & \\
\hline Reator forte & 1,4 & $0,5-3,8$ & 0,460 & 0,2 & $0,1-0,7$ & 0,011 \\
\hline Reator fraco & 0,6 & $0,1-5,0$ & 0,608 & 1,1 & $0,3-4,3$ & 0,969 \\
\hline Não realizado & 2,1 & $0,9-5,0$ & 0,098 & 1,2 & $0,6-2,4$ & 0,615 \\
\hline \multicolumn{7}{|l|}{ Sorologia para HIV } \\
\hline Negativo & 1,0 & & & 1,0 & & \\
\hline Positivo & 8,2 & $2,2-30,9$ & $<0,001$ & 4,1 & $0,7-24,0$ & 0,118 \\
\hline Em andamento & 1,5 & $0,5-4,5$ & 0,505 & 0,8 & $0,1-5,9$ & 0,785 \\
\hline Não realizado & 2,2 & $1,5-3,3$ & $<0,001$ & 1,8 & $1,1-2,9$ & 0,018 \\
\hline \multicolumn{7}{|l|}{ Tipo de entrada } \\
\hline Caso novo & 1,0 & & & 1,0 & & \\
\hline Retratamento & 2,4 & $1,3-4,5$ & 0,005 & 0,3 & $0,1-2,2$ & 0,237 \\
\hline \multicolumn{7}{|c|}{ Indicador de acompanhamento } \\
\hline Excelente & 1,0 & & & 1,0 & & \\
\hline Insuficiente & 11,1 & $5,2-24,8$ & $<0,001$ & 20,3 & $4,9-84,9$ & $<0,001$ \\
\hline Regular & 4,4 & $1,9-10,3$ & $<0,001$ & 5,1 & $1,2-22,7$ & 0,031 \\
\hline Bom & 1,5 & $0,5-4,9$ & 0,496 & 3,5 & $0,6-21,2$ & 0,181 \\
\hline
\end{tabular}

IC95\%: intervalo de 95\% de confiança; OR: odds ratio.

* Valor de $p<0,05$

foram registradas maiores chances de óbito. Por outro lado, os casos que tiveram reações fortes ao teste tuberculínico apresentaram menor chance de óbito $(\mathrm{OR}=0,2 ; \mathrm{IC} 95 \%$ : 0,1-0,7) (Tabela 4).

\section{Discussão}

Essa é a primeira vez que dados de notificação do SINAN são utilizados para investigar o perfil epidemiológico da TB entre crianças e adolescentes indígenas brasileiros. Por meio desta abordagem, obteve-se um panorama detalhado acerca do adoecimento e de fatores associados ao abandono e ao óbito na vigência do tratamento. Revelou-se ainda que crianças e adolescentes indígenas estão sujei- 
tos a elevadas incidências, com marcantes desigualdades regionais e diferenças expressivas segundo faixa etária.

Os maiores coeficientes de incidência concentraram-se nos adolescentes de 15-19 anos e nas crianças de 0-4 anos. Adicionalmente, os testes empregados para o diagnóstico de TB foram subutilizados, sobretudo os exames bacteriológicos. Foi possível ainda demonstrar que desfechos desfavoráveis ao tratamento, especialmente óbito e abandono, estiveram fortemente associados ao acompanhamento inadequado dos casos. Por fim, as crianças e adolescentes com manifestações extrapulmonares e mistas tiveram maiores chances de óbito.

Embora as incidências médias aqui reveladas (49,1/100 mil) pareçam próximas às reportadas na população geral do país, quando se comparam nossos achados com os descritos para crianças e adolescentes no Estado de São Paulo, onde Venâncio et al. 33 revelaram incidências de 3,2 e de 2,1/100 mil, entre 2001-2005 e 2006-2010, respectivamente, pode-se ter uma ideia da carga de adoecimento entre este segmento específico da população. Mesmo considerando países africanos, onde sabidamente há elevada magnitude de TB, Daniel et al. 34 e Wobudeya et al. 35 relatam incidências em torno de 17,0/100 mil e 44,0/100 mil na Nigéria e em Uganda, respectivamente, cifras inferiores às aqui reportadas.

Se considerarmos os dados desagregados por faixa etária e macrorregiões do país, as discrepâncias nos indicadores de incidência ficam ainda mais evidentes e demonstram a gravidade do problema, especialmente entre adolescentes e crianças menores de cinco anos, nas regiões Centro-oeste, Sudeste e Norte. Dados de magnitude semelhantes foram descritos somente por Gava et al. 22 e por Marques et al. 16, ao analisarem as notificações em crianças e adolescentes indígenas de Rondônia e Mato Grosso do Sul, nos períodos de 1997-2006 e 2000-2006, respectivamente.

As incidências aqui demonstradas são inferiores apenas às reportadas para população indígena adulta 5,10,11,13,14,24, para as quais a carga da doença é desproporcionalmente mais elevada em comparação a outros segmentos da sociedade. Parte dessa disparidade pode ser explicada pelas desigualdades sociais em saúde, que incluem acesso restrito à renda, emprego, educação formal e cuidados em saúde $36,37,38,39$.

O acesso restrito aos cuidados em saúde fica evidente na análise dos exames empregados no diagnóstico. Ao longo de todo período foi possível constatar que quase $1 / 4$ dos casos que foram submetidos a tratamento para TB não continham dados radiológicos conclusivos, quase $3 / 4$ não realizaram teste tuberculínico e quase metade não realizaram baciloscopias de escarro, considerados como os principais exames complementares. Ademais, cerca de $80 \%$ e $40 \%$ dos casos notificados não realizaram cultura e sorologia anti-HIV, respectivamente. Resultados semelhantes foram descritos no Amazonas e em Rondônia 10,22.

Embora empírico e não validado, o indicador de acompanhamento adotado neste estudo está fortemente ligado ao manejo dos casos de TB, no âmbito da atenção básica, uma vez que considera a realização de baciloscopias de controle no 2o, 4o e 6o meses de tratamento; o exame de contatos; e o tratamento diretamente observado. Em nossa interpretação, quando essas ações são realizadas de maneira regular e/ou insuficiente, o risco de abandono e óbito na vigência do tratamento é exacerbado, conforme apontado aqui e em outros estudos 10,24,25,26.

Quando nos dedicamos ao estudo dos fatores associados ao óbito e ao abandono, descobrimos que as crianças de 0-4 anos apresentaram risco aumentado de evoluir para óbito. Este fenômeno pode estar associado às dificuldades enfrentadas para o diagnóstico da TB na infância 40, sobretudo para os menores de dez anos, uma vez que os sinais e sintomas se confundem com outras doenças, e a coleta de amostras biológicas pode demandar exames de alta complexidade, como os lavados gástrico e broncoalveolar. Com a finalidade de contornar essa dificuldade, a análise combinada de dados clínicos (alterações radiológicas, vacinação BCG, resposta ao teste tuberculínico, estado nutricional) e epidemiológicos (contato com adultos bacilíferos) tem sido recomendada para reduzir os erros diagnósticos nas crianças. No Brasil, o Ministério da Saúde indica o uso do sistema de pontuação para o diagnóstico de TB na infância 41. Outra possibilidade reside no fato de as crianças apresentarem maior predisposição ao desenvolvimento de tuberculose primária extrapulmonar, especialmente as formas miliares e meníngea 18,42, que resultam em maior morbimortalidade 43,44.

Nesta análise, os casos que apresentaram reações fortes ao teste tuberculínico tiveram menor chance de óbito. Estudos realizados com diferentes grupos indígenas demonstraram elevadas 
proporções de não reatores ao teste tuberculínico, mesmo em locais onde a doença apresentava elevada incidência e a cobertura vacinal BCG excedia $80 \%$ 45,46. Longhi et al. 46 sugerem que a não -reatividade ao teste tuberculínico é decorrente de deficiências na resposta imune celular ou devida à susceptibilidade genética. Nessa perspectiva, nossos achados sugerem que as crianças e adolescentes indígenas que conseguiram desenvolver uma resposta forte ao teste tuberculínico podem ter tido melhores condições imunológicas para enfrentar a TB e, consequentemente, menor risco de evoluir para desfechos fatais.

Semelhantemente ao descrito em outros estudos com populações não indígenas da África do Sul 47 e de Camarões 48, em nossa análise, os casos em que o teste anti-HIV foi positivo ou não realizado apresentaram maior risco de abandono do tratamento. Ao menos em adultos, esse fenômeno pode ser atribuído ao estigma associado a aids e também ao medo de ser submetido a um excesso de medicamentos que usualmente são ofertados aos pacientes coinfectados em tratamento concomitante para TB. Essa associação leva a uma menor probabilidade de completar o tratamento 48 . Observamos ainda que crianças e adolescentes que entraram no SINAN para retratamento também apresentaram maior risco de abandono. O abandono é um sério problema para o controle da doença, pois o tratamento inadequado pode ser um dos principais determinantes da manutenção da cadeia de transmissão, favorecendo ainda o desenvolvimento de cepas resistentes às drogas 49 . Resultados semelhantes foram descritos por outros autores 10,50, ao identificarem aumento de abandono entre os casos de reingresso pós-tratamento na Região Amazônica. A presença de TB em crianças e adolescentes é um forte indicador da existência de transmissão ativa nas comunidades a partir de contato com adultos bacilíferos. As altas incidências reveladas neste estudo reforçam essa hipótese e comprovam transmissão ativa nas aldeias indígenas, sobretudo nas regiões Norte, Sudeste e Centro-oeste 16,23.

Pelo fato deste estudo ter se pautado em dados secundários de notificação, é importante mencionarmos algumas limitações. Por exemplo, a possibilidade de haver subregistro e/ou subnotificação de eventos, incompletude de algumas variáveis, erros na classificação e/ou codificação dos casos, sobretudo na variável cor ou raça 51. Em decorrência de termos incluído nas análises somente crianças e adolescentes, é oportuno considerar que os dados sobre cor ou raça não tenham seguido o critério de autoclassificação adotado pelo IBGE. Todavia, entendemos que as mulheres são as principais cuidadoras de crianças e adolescentes e que os profissionais ao preencherem as fichas de notificação podem ter adotado a cor ou raça da mãe/cuidadora. Em algumas circunstâncias pode realmente ter havido erro no preenchimento, mas acreditamos que esse erro seja de natureza aleatória e não tenha introduzido viés em nossas estimativas.

Em nossas análises não consideramos no cálculo de interpolação geométrica a taxa de crescimento anual segundo as categorias de residência urbana e rural, pois nossa unidade geográfica de análise foi macrorregião. Todavia, é admissível pensar que possa haver diferenças nos coeficientes de incidência de TB entre residentes em zonas urbanas e rurais. Outra limitação inclui a possibilidade de erros de diagnóstico nos casos de TB entre crianças indígenas já relatado na literatura ${ }^{23}$. Além disso, vale lembrar da impossibilidade de se obter dados sobre demografia, indicadores socioeconômicos e a respeito do estado nutricional, moradia, composição das famílias, a partir de consulta ao SINAN, fato que pode inserir confundimento residual nas estimativas. Por fim, é admissível supor que tenha ocorrido subnotificação de óbitos 52 e falhas nas estimativas dos denominadores populacionais 53 , fatos que podem ter gerado erro na classificação do desfecho e distorção nas estimativas de incidência.

Por outro lado, a abordagem aqui empregada tem representatividade nacional uma vez que incluiu na análise todos os casos de TB notificados em crianças e adolescentes indígenas no Brasil, considerando a variável cor ou raça, ao longo de mais de uma década. A escolha do modelo de regressão logística multinomial permitiu identificar simultaneamente os fatores associados ao abandono e ao óbito na vigência do tratamento, a partir de variáveis clínico-epidemiológicas. Esta abordagem não foi identificada em estudos anteriores sobre a temática.

Acreditamos que o controle da TB em crianças e adolescentes indígenas não poderá ser alcançado sem investimentos em pesquisa e desenvolvimento e sem a redução das desigualdades sociais. Há urgente necessidade de aprimorar as ferramentas de diagnóstico e garantir o acesso às ferramentas existentes. As opções terapêuticas para crianças precisam ser expandidas, bem como as ações de prevenção que incluem investigação de contatos, diagnóstico e tratamento da infecção tuberculosa latente e ampliação da vacinação BCG em tempo oportuno, além do diagnóstico precoce e tratamento 
imediato das pessoas com TB ativa 54,55 . Somente dessa maneira se vislumbrará uma redução na carga de TB entre crianças e adolescentes indígenas no Brasil. Esforços contínuos para o controle de contatos devem ser intensificados para prevenir a transmissão com o objetivo de acabar com a TB como problema de saúde pública no país e no mundo até o ano de 2035.

\section{Colaboradores}

P. V. S. Viana e P. C. Basta participaram da concepção do estudo, analisaram os dados e participaram da elaboração e revisão final do texto. S. B. Codenotti e A. L. Bierrenbach participaram da concepção do estudo e revisão final do texto.

\section{Informações adicionais}

ORCID: Paulo Victor de Sousa Viana (0000-00028449-2705); Stefano Barbosa Codenotti (00000002-6862-5950); Ana Luiza Bierrenbach (00000002-6837-0636); Paulo Cesar Basta (0000-00030804-0413).

\section{Referências}

1. Montenegro RA, Stephens C. Indigenous health in Latin America and the Caribbean. Lancet 2006; 367:1859-69.

2. Romero-Sandoval NC, Flores-Carrera OF, Sánchez-Pérez HJ, Sánchez-Pérez I, Mateo MM. Pulmonary tuberculosis in an indigenous community in the mountains of Ecuador. Int J Tuberc Lung Dis 2007; 11:550-5.

3. Giuffrida A. Racial and ethnic disparities in Latin America and the Caribbean : a literature review. Divers Equal Health Care 2010; 7:11529.

4. Coimbra Jr. CE, Santos RV, Welch JR, Cardoso AM, de Souza MC, Garnelo L, et al. The First National Survey of Indigenous People's Health and Nutrition in Brazil: rationale, methodology, and overview of results. BMC Public Health 2013; 13:52.

5. Basta PC, Orellana JDY, Arantes R. Perfil epidemiológico dos povos indígenas no Brasil: notas sobre agravos selecionados. In: Garnelo L, Pontes AL, organizadores. Saúde indígena: uma introdução ao tema. 2a Ed. Brasília: Ministério da Saúde; 2012. p. 60-106.

6. Cardoso AM, Santos RV, Coimbra Jr. CEA. Mortalidade infantil segundo raça/cor no Brasil: o que dizem os sistemas nacionais de informação? Cad Saúde Pública 2005; 21:1602-8.

7. Pan WK, Erlien C, Bilsborrow RE. Morbidity and mortality disparities among colonist and indigenous populations in the Ecuadorian Amazon. Soc Sci Med 2010; 70:401-11.

\section{Agradecimentos}

Os autores agradecem ao Programa Nacional de Controle da Tuberculose pela disponibilização dos dados de notificação de tuberculose no Brasil, em especial à Coordenadora-Geral Denise ArakakiSanchez, a Patricia Bartholomay Oliveira e a Daniele Maria Pelissari.
8. Horta BL, Santos RV, Welch JR, Cardoso AM, Dos Santos JV, Assis AM, et al. Nutritional status of indigenous children: findings from the First National Survey of Indigenous People's Health and Nutrition in Brazil. Int J Equity Health 2013; 12:23.

9. Melo TEMP, Resendes APC, Souza-Santos R, Basta PC. Distribuição espacial e temporal da tuberculose em indígenas e não indígenas de Rondônia, Amazônia Ocidental, Brasil. Cad Saúde Pública 2012; 28:267-80.

10. Belo EN, Douglas J, Orellana Y, Levino A, Basta C. Tuberculose nos municípios amazonenses da fronteira Brasil-Colômbia-Peru-Venezuela: situação epidemiológica e fatores associados ao abandono. Rev Panam Salud Pública 2013; 34:321-9.

11. Basta PC, Oelemann MAC, Oelemann WMR, Fonseca LS, Coimbra Jr. CEA. Detection of Mycobacterium tuberculosis in sputum from Surui Indian subjects, Brazilian Amazon. Mem Inst Oswaldo Cruz 2006; 101:581-4.

12. Malacarne J, Rios DPG, da Silva CMFP, Braga JU, Camacho LAB, Basta PC. Prevalence and factors associated with latent tuberculosis infection in an indigenous population in the Brazilian Amazon. Rev Soc Bras Med Trop 2016; 49:456-64.

13. Rios DPG, Malacarne J, Alves LCC, Sant'Anna CC, Camacho LAB, Basta PC. Tuberculose em indígenas da Amazônia brasileira: estudo epidemiológico na região do Alto Rio Negro. Rev Panam Salud Pública 2013; 33:22-9. 
14. Basta P, Coimbra Jr. CEA, Camacho LAB, Santos R. Risk of tuberculosis infection in an indigenous population from Amazonia, Brazil. Int J Tuberc Lung Dis 2006; 10:1354-9.

15. Jenkins HE. Global burden of childhood tuberculosis. Pneumonia (Nathan) 2016; 8:24.

16. Marques AMC, Pompilio MA, Santos SC, Garnes SJA, Cunha RV. Tuberculose em indígenas menores de 15 anos, no Estado de Mato Grosso do Sul. Rev Soc Bras Med Trop 2010; 43:700-4.

17. Basta PC, Coimbra Jr. CEA, Escobar AL, Santos RV. Aspectos epidemiológicos da tuberculose na população indígena Suruí, Amazônia, Brasil. Rev Soc Bras Med Trop 2004; 37:33842.

18. Perez-Velez CM, Marais BJ. Tuberculosis in children. N Engl J Med 2012; 367:348-61.

19. Ruiz Jiménez M, Guillén Martín S, Prieto Tato LM, Cacho Calvo JB, Álvarez García A, Soto Sánchez B, et al. Induced sputum versus gastric lavage for the diagnosis of pulmonary tuberculosis in children. BMC Infect Dis 2013; 13:222.

20. Coordenação-Geral de Desenvolvimento da Epidemiologia em Serviços, Secretaria de Vigilância em Saúde, Ministério da Saúde. Guia de vigilância em saúde. v. 2. Brasília: Ministério da Saúde; 2017.

21. Sant'Anna CC, Orfaliais CTS, De March MFP, Conde MB. Evaluation of a proposed diagnostic scoring system for pulmonary tuberculosis in Brazilian children. Int J Tuberc Lung Dis 2006; 10:463-5.

22. Gava C, Malacarne J, Rios DPG, Sant'Anna CC, Camacho LAB, Basta PC. Tuberculosis in indigenous children in the Brazilian Amazon. Rev Saúde Pública 2013; 47:77-85.

23. Basta PC, Rios DPG, Alves LCC, Sant'Anna CC, Coimbra Jr. CEA. Estudo clínico-radiológico de crianças e adolescentes indígenas Suruí, Região Amazônica. Rev Soc Bras Med Trop 2010; 43:719-22.

24. Orellana JDY, Gonçalves MJF, Basta PC. Características sociodemográficas e indicadores operacionais de controle da tuberculose entre indígenas e não indígenas de Rondônia, Amazônia Ocidental, Brasil. Rev Bras Epidemiol 2012; 15:846-56.

25. Basta PC, Marques M, Oliveira RL, Cunha EAT, Resendes APC, Souza-Santos R. Desigualdades sociais e tuberculose: análise segundo raça/cor, Mato Grosso do Sul Social. Rev Saúde Pública 2013; 47:854-64.

26. Viana PVS, Gonçalves MJF, Basta PC. Ethnic and racial inequalities in notified cases of tuberculosis in Brazil. PLoS One 2016; 11:e0154658.

27. Conde MB, Melo FAF, Marques AMC, Cardoso NC, Pinheiro VGF, Dalcin PDTR, et al. III Diretrizes para Tuberculose da Sociedade Brasileira de Pneumologia e Tisiologia. J Bras Pneumol 2009; 35:1018-48.

28. Ministério da Saúde. Portaria no 3.947, de 25 de novembro de 1998. Diário Oficial da União 1999; 14 jan.
29. Givisiez GHN. Introdução à métodos de estimativas e interpolações populacionais. In: Rios-Neto ELG, Riani JLR, organizadores. Introdução à demografia da educação. v. 1. Campinas: Associação Brasileira de Estudos Populacionais; 2004. p. 45-70.

30. Hosmer DW, Lemeshow S, Sturdivant RX. Applied logistic regression. Hoboken: John Wiley \& Sons; 2013.

31. Fagerland MW, Hosmer DW, Bofin AM. Multinomial goodness-of-fit tests for logistic regression models. Stat Med 2008; 27:4238-53.

32. Brasil. Lei no 12.527, de 18 de novembro de 2011. Regulamenta o direito constitucional de acesso às informações públicas. Diário Oficial da União 2011; 18 nov.

33. Venâncio TS, Tuan TS, Nascimento LFC. Incidence of tuberculosis in children in the state of São Paulo, Brazil, under spatial approach. Ciênc Saúde Colet 2015; 20:1541-7.

34. Daniel OJ, Adejumo OA, Abdur-Razzaq HA, Ebunoluwa JO. Trend of childhood TB case notification in Lagos, Nigeria, 2011-2014. Int J Mycobacteriol 2015; 4:239-44.

35. Wobudeya E, Lukoye D, Lubega IR, Mugabe F, Sekadde M, Musoke P. Epidemiology of tuberculosis in children in Kampala district, Uganda, 2009-2010; a retrospective cross-sectional study. BMC Public Health 2015; 15:967.

36. Coimbra Jr. CEA, Basta PC. The burden of tuberculosis in indigenous peoples in Amazonia, Brazil. Trans R Soc Trop Med Hyg 2007; 101:635-6

37. Ring I, Brown N. The health status of indigenous peoples and others. BMJ 2003; 327:4045.

38. Bloss E, Holtz TH, Jereb J, Redd JT, Podewils $\mathrm{LJ}$, Cheek JE, et al. Tuberculosis in indigenous peoples in the U.S., 2003-2008. Public Health Rep 2011; 126:677-89.

39. Coimbra Jr. CEA, Santos RV. Saúde, minorias e desigualdade: algumas teias de inter-relações, com ênfase nos povos indígenas no Brasil. Ciênc Saúde Colet 2000; 5:125-32.

40. Jenkins HE, Yuen CM, Rodriguez CA, Nathavitharana RR, McLaughlin MM, Donald $\mathrm{P}$, et al. Mortality in children diagnosed with tuberculosis: a systematic review and metaanalysis. Lancet Infect Dis 2017; 17:285-95.

41. Ministério da Saúde. Manual de recomendações para o controle da tuberculose no Brasil. Brasília: Ministério da Saúde; 2011.

42. Maltezou HC, Spyridis P, Kafetzis DA. Extrapulmonary tuberculosis in children. Arch Dis Child 2000; 83:342-6.

43. Marais BJ, Gie RP, Schaaf HS, Hesseling AC, Obihara CC. The natural history of childhood intra-thoraci tuberculosis: a critical review of literature from the pre-chemotherapy era. Int J Tuberc Lung Dis 2004; 8:392-402.

44. Marais BJ, Gie RP, Schaaf HS, Hesseling AC, Enarson DA, Beyers N. The spectrum of disease in children treated for tuberculosis in a highly endemic area. Int J Tuberc Lung Dis 2006; 10:732-8. 
45. Zembrzuski VM, Basta PC, Callegari-Jacques SM, Santos RV, Coimbra Jr. CEA, Salzano FM, et al. Cytokine genes are associated with tuberculin skin test response in a native Brazilian population. Tuberculosis 2010; 90:44-9.

46. Longhi RMP, Zembrzuski VM, Basta PC, Croda J. Genetic polymorphism and immune response to tuberculosis in indigenous populations: a brief review. Braz J Infect Dis 2013; 17:363-8.

47. Kigozi G, Heunis C, Chikobvu P, Botha S, van Rensburg D. Factors influencing treatment default among tuberculosis patients in a high burden province of South Africa. Int J Infect Dis 2017; 54:95-102.

48. Pefura Yone EW, Kuaban C, Kengne AP. HIV testing, HIV status and outcomes of treatment for tuberculosis in a major diagnosis and treatment centre in Yaounde, Cameroon: a retrospective cohort study. BMC Infect Dis 2012; 12:190.

49. Toczek A, Cox H, Du Cros P, Cooke G, Ford N. Strategies for reducing treatment default in drug-resistant tuberculosis: systematic review and meta-analysis. Int J Tuberc Lung Dis 2013; 17:299-307.

50. Garrido MS, Penna ML, Perez-Porcuna TM, de Souza AB, Marreiro LS, Albuquerque BC, et al. Factors associated with tuberculosis treatment default in an endemic area of the Brazilian Amazon: a case control-study. PLoS One 2012; 7:e39134.
51. Laguardia J, Domingues CMA, Carvalho C, Lauerman CR, Macário E, Glatt R. Sistema de Informação de Agravos de Notificação (Sinan): desafios no desenvolvimento de um sistema de informação em saúde. Epidemiol Serv Saúde 2004; 13:135-47.

52. Oliveira GP, Pinheiro RS, Coeli CM, Barreira D, Codenotti SB. Uso do sistema de informação sobre mortalidade para identificar subnotificação de casos de tuberculose no Brasil. Rev Bras Epidemiol 2012; 15:468-77.

53. Bastos JL, Santos RV, Cruz OG, Longo LAFB, Silva LO. Características sociodemográficas de indígenas nos censos brasileiros de 2000 e 2010: uma abordagem comparativa. Cad Saúde Pública 2017; 33 Suppl 1:e00085516.

54. World Health Organization. BCG vaccine: WHO position paper, February 2018 - recommendations. Vaccine 2018; 93:73-96.

55. Marais BJ. Improving access to tuberculosis preventive therapy and treatment for children. Int J Infect Dis 2017; 56:122-5. 


\section{Abstract}

The study aimed to describe clinical and sociodemographic characteristics, estimate incidence, and analyze factors associated with dropout and death during treatment of $T B$ cases reported in indigenous children and adolescents in Brazil from 2006 to 2016. A historical case series was performed on incidence according to age bracket and major geographic region, and multinomial logistic regression was used to explain factors associated with treatment dropout and death. Of the 2,096 reported cases, $88.2 \%$ evolved to cure, $7.2 \%$ dropped out of treatment, and $4.6 \%$ evolved to death. There was a predominance of cases in boys 15-19 years of age and a higher proportion of deaths (55.7\%) in children < 4 years. Considering indigenous children and adolescents with TB in Brazil as a whole, mean incidence was 49.1/100,000, ranging from 21.5/100,000 to 97.6/100,000 in the Northeast and Central, respectively. Cases with insufficient and irregular follow-up showed higher odds of dropout $(\mathrm{OR}=11.1 ; 95 \% \mathrm{CI}: 5.2-24.8 / \mathrm{OR}=4.4$; 95\% CI: 1.9-10.3) and death $(\mathrm{OR}=20.3$; 95\% CI: 4.9-84.9/OR = 5.1; 95\% CI: 1.2-22.7). Cases in retreatment $(O R=2.4 ; 95 \% C I: 2.08-8.55)$ and with HIV coinfection (OR = 8.2; 95\% CI: 2.2-30.9) were also associated with dropout. Extrapulmonary $(\mathrm{OR}=1.8 ; 95 \% \mathrm{CI}: 1.1-3.3)$ and mixed clinical forms $(\mathrm{OR}=5.6$; 95\%CI: 2.8-11.4), age < 4 years $(O R=3.1 ; 95 \% C I: 1.5-6.4)$, and cases from the North $(\mathrm{OR}=2.8 ; 95 \% \mathrm{CI}: 1.1-7.1)$ and Central $(O R=2.8 ; 95 \%$ CI: 1.1-7.0) were associated with death. TB control in indigenous children and adolescents cannot be achieved without investments in research and development and without reducing social inequalities.

Tuberculosis; South American Indians; Death; Patient Dropouts

\section{Resumen}

El objetivo de este estudio fue describir características clínicas y sociodemográficas, estimar la incidencia de la tuberculosis (TB), además de analizar factores asociados al abandono y al óbito en la vigencia del tratamiento de los casos de TB, notificados entre niños y adolescentes indígenas, en Brasil entre 2006-2016. Se realizó un análisis de la serie histórica de incidencia, según la franja de edad y macrorregión y se utilizó la regresión logística multinomial para elucidar factores asociados al abandono y al óbito. Del total de 2.096 casos notificados, un $88,2 \%$ tuvieron cura, un 7,2\% abandonaron el tratamiento y un 4,6\% evolucionaron hacia óbito. Hubo un predominio de casos en chicos de 15-19 años y mayor proporción de óbitos $(55,7 \%)$ en < 4 años. Considerando el conjunto de niños y adolescentes indígenas con TB en Brasil, la incidencia media fue 49,1/100.000, variando de 21,5/100.000 a 97,6/100.000 en las regiones Nordeste y Centro-oeste, respectivamente. Los casos con un seguimiento insuficiente y regular tuvieron mayores oportunidades de abandono $(\mathrm{OR}=11,1$; IC95\%: 5,2-24,8/OR = 4,4; IC95\%: 1,9-10,3) y óbito $(\mathrm{OR}=20,3$; IC95\%: 4,9-84,9/OR = 5, 1; IC95\%: 1,2-22,7). Los casos de retorno al tratamiento $(\mathrm{OR}=2,4$; IC95\%: 2,08-8,55) y con antiVIH positivo (OR = 8,2; IC95\%: 2,2-30,9) también se mostraron asociados al abandono. Las formas clínicas extrapulmonares $(O R=1,8$; IC95\%: 1,13,3) y mixta (OR $=5$, 6; IC95\%: 2,8-11,4), los casos en < 4 años $(O R=3,1$; IC95\%: 1,5-6,4) y los casos procedentes de las regiones Norte $(\mathrm{OR}=2,8$; IC95\%: 1,1-7,1) y Centro-oeste (OR = 2, 8; IC95\%: 1,1-7,0) se mostraron asociados al óbito. Creemos que el control de la TB en niños y adolescentes indígenas no se podrá alcanzar sin inversiones en investigación y desarrollo y sin la reducción de las desigualdades sociales.

Tuberculosis; Indios Sudamericanos; Muerte; Pacientes Desistentes del Tratamiento
Recebido em 17/Abr/2018

Versão final reapresentada em 14/Dez/2018 Aprovado em 11/Jan/2019 